\title{
Analysis of the relationship between end-to-end distance and activity of single-chain antibody against colorectal carcinoma
}

\author{
Jianhua Zhang ${ }^{1 \dagger}$, Shanhong Liü ${ }^{2 \dagger}$, Zhigang Shang ${ }^{1}$, Li Shi ${ }^{{ }^{*}}$ and Jun Yun ${ }^{3^{*}}$
}

\author{
*Correspondence: shi_li2012@126. \\ com; jun_yun2000@126.com \\ ${ }^{\dagger}$ Equal contributors \\ ${ }^{1}$ Faculty of Biomedical Engineering \\ of Zhengzhou University, \\ Zhengzhou 450001, Henan \\ Province, People's Republic of China \\ ${ }^{3}$ Vascular Endocrinology \\ Department of Xijing Hospital \\ Affiliated to the Fourth Military \\ Medical University, Xi'an 710032, \\ Shaanxi Province, People's Republic \\ of China \\ Full list of author information is \\ available at the end of the article
}

\begin{abstract}
We investigated the relationship of End-to-end distance between $\mathrm{VH}$ and $\mathrm{VL}$ with different peptide linkers and the activity of single-chain antibodies by computer-aided simulation. First, we developed $\left(G_{4} S\right)_{n}$ (where $n=1-9$ ) as the linker to connect $\mathrm{VH}$ and $\mathrm{VL}$, and estimated the 3D structure of single-chain FV antibody (scFv) by homologous modeling. After molecular models were evaluated and optimized, the coordinate system of every protein was built and unified into one coordinate system, and End-to-end distances calculated using 3D space coordinates. After expression and purification of scFv-n with $\left(G_{4} S\right) n$ as $n=1,3,5,7$ or 9, the immunoreactivity of purified ND-1 scFv- $n$ was determined by ELISA. A multi-factorial relationship model was employed to analyze the structural factors affecting scFv: $r(n)=\sqrt{\left[A B(n)-\mathrm{A} B_{0}\right]^{2}+\left[C D(n)-C D_{0}\right]^{2}}+\left[B C(n)-B C_{s t}\right]^{2}$. The relationship between immunoreactivity and $r$-values revealed that fusion protein structure approached the desired state when the $r$-value $=3$. The immunoreactivity declined as the $r$-value increased, but when the $r$-value exceeded a certain threshold, it stabilized. We used a linear relationship to analyze structural factors affecting scFv immunoreactivity.
\end{abstract}

Keywords: Single-chain Fv antibody (scFv), (Gly $\left.{ }_{4} \mathrm{Ser}\right) \mathrm{n}$, End-to-end distance, Homologous modeling, Meta MQAP

\section{Biomed Central}

\section{Introduction}

Single-chain $\mathrm{Fv}$ antibody ( $\mathrm{scFv}$ ) is composed of immunoglobulin heavy- and lightchain variable regions connected by a short peptide linker [1-3]. ScFv is an ideal tool for the construction of single-chain bi-specific antibody fusion proteins [4-6]. Bivalent antibodies derived from $\mathrm{scFv}$ using genetic engineering have a promising future in the clinic. scFvs can be therapeutic and at the same time serve as a vector for delivering a toxin [7]. In recent years, there has been progress in colorectal cancer diagnosis and treatment using $\mathrm{scFv}$ as a carrier. However, achieving both high affinity and anti-tumor activity can be difficult, particularly since both are needed to be effective. Studies have shown that a proper linker can provide a $\mathrm{scFv}$ with biological activity more effective for clinical applications [8-10]. Consequently, choosing and designing a proper linker is a key consideration.

(c) 2012 Zhang et al.; licensee BioMed Central Ltd. This is an Open Access article distributed under the terms of the Creative Commons Attribution License (http://creativecommons.org/licenses/by/2.0), which permits unrestricted use, distribution, and reproduction in any medium, provided the original work is properly cited. 
Proteomics has revealed a great deal about the composition, structure, and function of proteins, and bioinformatics provides a powerful tool to study the structure-activity relationship of fusion proteins [11-13]. Drug design based on structural simulation incorporates 3D structure, including data from fusion proteins with various functional domains and inter-peptide linkers [14-16]. Linkers that contain $\left(\mathrm{G}_{4} \mathrm{~S}\right) \mathrm{n}$ are the most widely used [12,17], prompting us to examine its effects on the structure and function of scFvs.

\section{Materials and methods}

\section{Materials}

IC-2 and CCL-187 cells were cultured using standard conditions. IC-2 is a murine hybridism cell line that secretes the monoclonal antibody ND-1, specific for human colorectal carcinoma. CCL-187 is a human colorectal carcinoma cell line. The pET28a (+) expression vector and E. coli BL21 were contributed by Prof. J. Yun, Xi'an (China). The pMD18-T vector, E.coli JM109 competent cells, DNA polymerase, restriction enzymes, and DNA recovery kits were purchased from TaKaRa Biotechnology (Shanghai, China). mRNA purification kits and T4 DNA ligase were purchased from Pharmacia Biotech (Shanghai, China). Anti-His6 tag antibody was obtained from Invitrogen (Foster City, CA, USA). Ni-NTA resin was provided by QIAGEN (Shanghai, China), MDP and 99mTc were kindly provided by the Department of Nuclear Medicine of China Medical University (Liaoning Province, China). Heavy chain primer 1 and 2, light chain primer mix, linkers [(GGGGS)n] primer mix, and RS primer mix were purchased from Pharmacia Biotech.

ND-1 scFv-n was constructed as previously described. Briefly, mRNA was extracted from $5 \times 10^{6}$ IC-2 hybridism cells and cDNA synthesized by reverse transcription using random primers. $\mathrm{VH}$ and $\mathrm{VL}$ genes were separately amplified from cDNA by PCR using a heavy and light chain primer mix. The VH and VL gene fragments were recovered and mixed in equimolar ratios for two PCR reactions, with the first one using a linker primer mix for 7 cycles, followed by a second one using a RS primer mix for 30 cycles. As a result, $\mathrm{VH}$ and VL gene fragments were linked to form a scFv construct by extension, with overlapping splicing PCR. The resulting ND-1 scFv-n construct was cloned into pMD18-T and transformed into E. coli JM109, and positive clones identified by colony PCR and DNA sequencing.

Oligonucleotide primers S1 and S2 were designed to add EcoRI sites at the 5'-end of ND-1scFv-n, and a HindIII site, or SalI site at the 3'-end. S1: 5'-CTGAATTCATGGCC CAGGTGCAGCTGCAGC-3'; S2: 5'-CGCAAGCTTCTAGTCGACTTTCCAGCTTG GTC-3'. pMD18-T-ND-1scFv-n was used as a template, and the product cloned into the vector pET28a $(+)$ after digestion with EcoRIand HindIII, and transformed into competent E.coli BL21 cells for protein expression.

\section{Amino acid sequence}

The amino acid sequence of the wild-type $\mathrm{VH}$ and wild-type $\mathrm{VL}$ are listed below [18], and illustrated in Figure 1. The amino acid sequence of the VH- $\left(\mathrm{G}_{4} \mathrm{~S}\right) \mathrm{n}-\mathrm{VL}$ is: 


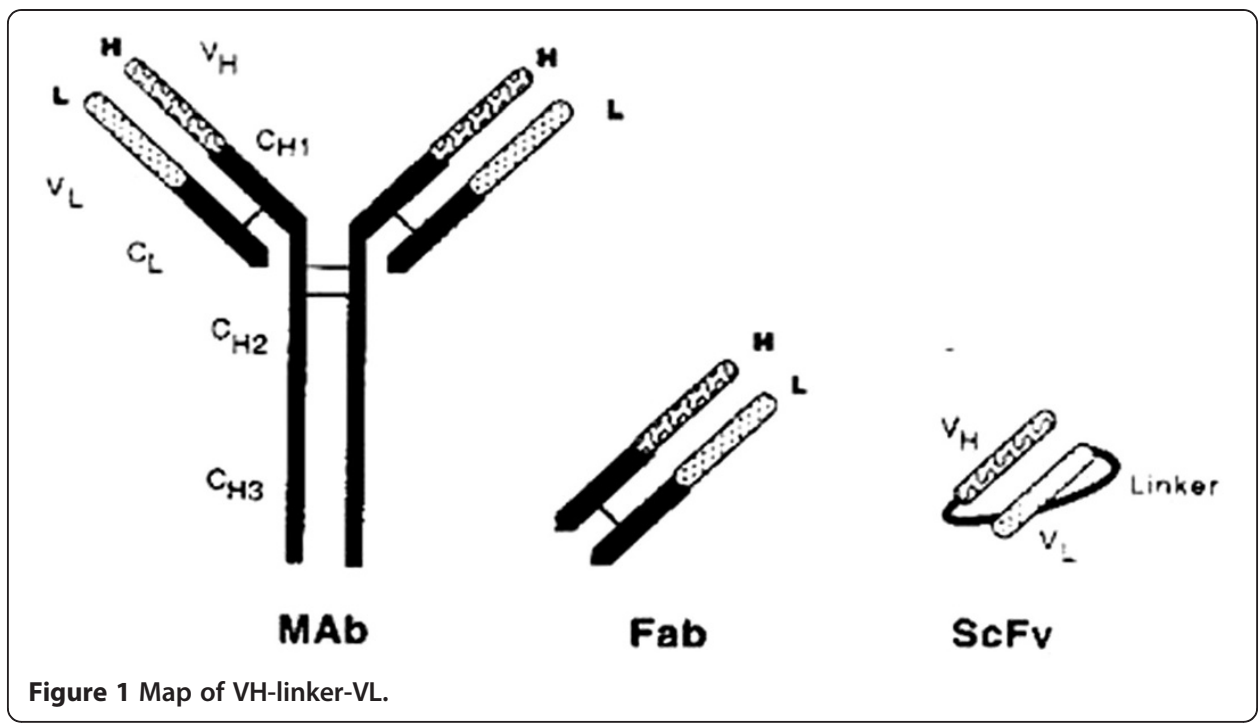

QGTTVTVSS(GGGGS)nDIELTQSPASLAVSLGQRATISYRASKSVSTSGYSYMHWQQ KPGQPPRLLIYLVSNLESGVPARFSGSGSGTDFTLNIHPVEEEDAATYYCQHIRELTRSE GGPSWK.

\section{Homology modeling, assessment, and optimization}

The amino acid sequence of a protein determines its high-level structure. Determining high-level protein structure relies on the identification of one or more known protein "templates" that resemble the structure of the query sequence, and alignment of the query sequence residues to the template residues. Swiss-Models can be used for homology modeling to search protein sequence and structure databases, such as the Protein Data Bank (PDB) [19-21]. A three-dimensional model of the targeted molecule can be obtained through homology modeling, and used to assess and optimize the model using Meta MQAP [22,23].

\section{Construction of coordinate system}

PDB files were obtained from Swiss-Model with the videotext coordinate system (in which the atomic coordinates are located), in order to facilitate protein structure comparison. The coordinate systems were constructed with Matlab7.0.

\section{Determination of the origin of the coordinate system}

The molecular weight of the atoms in the protein was used to calculate molecular weight, and the centric was obtained using the atomic location of each atom. The centric is the origin of the new coordinate system [24].

$$
\left[X_{0}, Y_{0}, Z_{0}\right]=\left[\frac{1}{N} \sum_{k=1}^{N} \frac{X_{k} * m_{k}}{M}, \frac{1}{N} \sum_{k=1}^{N} \frac{Y_{k} * m_{k}}{M}, \frac{1}{N} \sum_{k=1}^{N} \frac{Z_{k} * m_{k}}{M}\right]
$$

$\mathrm{N}$ : the number of all the atoms; $\mathrm{M}=12.01+14.01+16.00+32.07+1.00$;

Mi: molecular weight of atoms;

$\left[\mathrm{X}_{\mathrm{k}}, \mathrm{Y}_{\mathrm{k}}, \mathrm{Z}_{\mathrm{k}}\right]$ : the original three-dimensional coordinates of atoms;

$\left[\mathrm{X}_{0}, \mathrm{Y}_{0}, \mathrm{Z}_{0}\right]$ : the origin of the new coordinate system. 
To determine axes we constructed a second-order moment matrix of the protein's atomic coordinates. This was regarded as the principal component of the matrix's eigenvector of the new coordinate system's X-axis, the sub-principal component of the vector Y-axis, and used to build a coordinate system of the protein's three-dimensional structure.

The $3 \times 3$ matrix constructed by the second-order moment matrix is as follows:

$$
S=\left[\begin{array}{lll}
M_{200} & M_{110} & M_{101} \\
M_{110} & M_{020} & M_{011} \\
M_{101} & M_{011} & M_{002}
\end{array}\right]
$$

Here, $M_{a b c}=\sum_{k} m_{k} \times X_{k}^{a} \times Y_{k}^{b} \times Z_{k}^{c}$,

$\mathrm{m}_{\mathrm{k}}$ : molecule weight of atoms.

$\left[\mathrm{X}_{\mathrm{k}}, \mathrm{Y}_{\mathrm{k}}, \mathrm{Z}_{\mathrm{k}}\right]$ : 3D coordinates of each atom.

The eigenvalues and eigenvectors of $S$ were calculated, and the eigenvector calculated corresponding to the maximum eigenvalue as the first axis ( $\mathrm{X}$ axis is set, $\mathrm{X}=[\mathrm{X} 1, X 2$, $\mathrm{X} 3]$ ), with the eigenvector corresponding to the second largest eigenvalue as the second axis ( $\mathrm{Y}$ axis set, $\mathrm{Y}=[\mathrm{Y} 1, \mathrm{Y} 2, \mathrm{Y} 3])$, and similarly for the $\mathrm{Z}$ axis.

\section{Analysis of End-to-end distance in fusion proteins}

The End-to-end distance is the distance between the first and the last $\alpha$-carbon atom in a protein. We obtained this information and the $\mathrm{X} / \mathrm{Y} / \mathrm{Z}$ coordinates of the atoms from the PDB database. The algorithm used is as follows:

A. Locate the first and last $\alpha$-carbon atoms in the wild-type $\mathrm{VH}$ and $\mathrm{VL}$, and the same in the protein after introduction of $\left(\mathrm{G}_{4} \mathrm{~S}\right) \mathrm{n}$.

B. Calculate End-to-end distance of wild-type VH (VL) and mutant VH (VL) after introduction of $\left(\mathrm{G}_{4} \mathrm{~S}\right) \mathrm{n}$.

C. Analyze the relationship between the End-to-end distance and $n$.

\section{Biological experiments}

Expression and purification of ND-1scFv- $n$.

pET28a(+)-ND-1scFv-n plasmids were constructed as expression vectors and transformed into E. coli BL21 cells, which were grown in $100 \mathrm{ml} \mathrm{LB}$ broth with $50 \mathrm{mg} / \mathrm{ml}$ Kanamycin at $37^{\circ} \mathrm{C}$. When the culture attained an O.D. of 0.6, IPTG was added to a final concentration of $1 \mathrm{mM}$, and cells were shaken at $37^{\circ} \mathrm{C}$. After $3.5 \mathrm{~h}$, the culture was centrifuged at 5,000 rpm for $10 \mathrm{~min}$, and the cell pellets treated with lysis solution. After sonication and centrifugation, inclusion bodies containing scFv proteins were solubilized and denatured in the presence of $6 \mathrm{M}$ guanidine hydrochloride. Affinity chromatography on Ni-NTA resin was use to purify scFv, and the column eluted sequentially with $8 \mathrm{M}$ urea at pH8.0, 6.5 and 4.2. The pH4.2 fraction, containing scFv, was collected and recaptured by dialysis. Protein purity and concentration were determined by Bradford assay.

\section{Western blot analysis}

ND-1scFv- $n$ proteins were detected by western blot analysis. BL21 transformed with pET-28a(+)ND-1scFv- $n$ was incubated separately in loading buffer $(125 \mathrm{mmol} / \mathrm{L}$ Tris$\mathrm{HCl}, \mathrm{pH} 6.8,10 \% \beta$-mercapto-ethanol, 4.6\% SDS, $20 \%$ glycerol and $0.003 \%$ bromophenol blue) for $5 \mathrm{~min}$ at $100^{\circ} \mathrm{C}$, separated by sodium dodecyl sulfate polyacrylamide gel 
(SDS-PAGE), and electro blotted onto PVDF membrane (Bio-Rad, Hercules, CA, USA). Non-specific binding sites were blocked for $1 \mathrm{~h}$ with $5 \%$ nonfat milk in TPBS (PBS contained $0.05 \%$ Twin 20), and the membrane incubated overnight at $4{ }^{\circ} \mathrm{C}$ with primary antibody. After washing $3 \mathrm{X}$ in TPBS, the membrane was incubated with horseradish peroxidase-conjugated goat anti-rabbit IgG for $2 \mathrm{~h}$ at room temperature, and washed $2 \mathrm{X}$ with TPBS. Immunoblot signal was detected by autoradiography using an enhanced chemiluminescence detection kit.

\section{ELISA assay for activity of ND-1scFv-n}

CCL-187 cells $\left(5 \times 10^{4}\right)$ were grown in 96 -well micro titer plates at $37^{\circ} \mathrm{C}$ for $24 \mathrm{~h}$, fixed with $2.5 \%$ glutaraldehyde and blocked with $1 \%$ BSA, followed by incubation with ND1 IgG or ND-1scFv at $37^{\circ} \mathrm{C}$ for $2 \mathrm{~h}$. After washing $3 \mathrm{X}$ with PBS, anti-His6 antibody was added to wells with ND-1scFv-n and incubated. The plate was washed and HRP-labeled goat anti-mouse IgG was added into both ND-IgG and ND-1scFv wells. After incubating at $37^{\circ} \mathrm{C}$ for $2 \mathrm{~h}$, TMB substrate was added, and samples incubated in darkness for $30 \mathrm{~min}$. The reaction was terminated with $1 \mathrm{M} \mathrm{H}_{2} \mathrm{SO}_{4}$. PBS was used as a negative control.

\section{Results}

\section{Protein structures}

A videotext of the coordinate system was built using the PDB atomic coordinates from PDB files received from SWISS-MODEL, using Mat lab 7.0. The maps were used for comparison of the protein structures (Figure 2). Homology modeling using SWISS-MODEL was used to evaluate the best evaluation method. Meta-MQAP was used to assess and optimize the model. The accuracy score of the model and the root mean square (RMS) deviation are shown in Table 1. The assessment result shows that the model is reliable.

\section{Local alignment}

The End-to-end distance of VH (AB), VL (CD) and linker (BC), at different $n$ values are presented in Table 2. It appears that linker $\mathrm{BC}$ was relatively stable from $n=1-7$, and there were changes in the End-to-end distances for $\mathrm{AB}$ and $\mathrm{CD}$. When the $n$ value

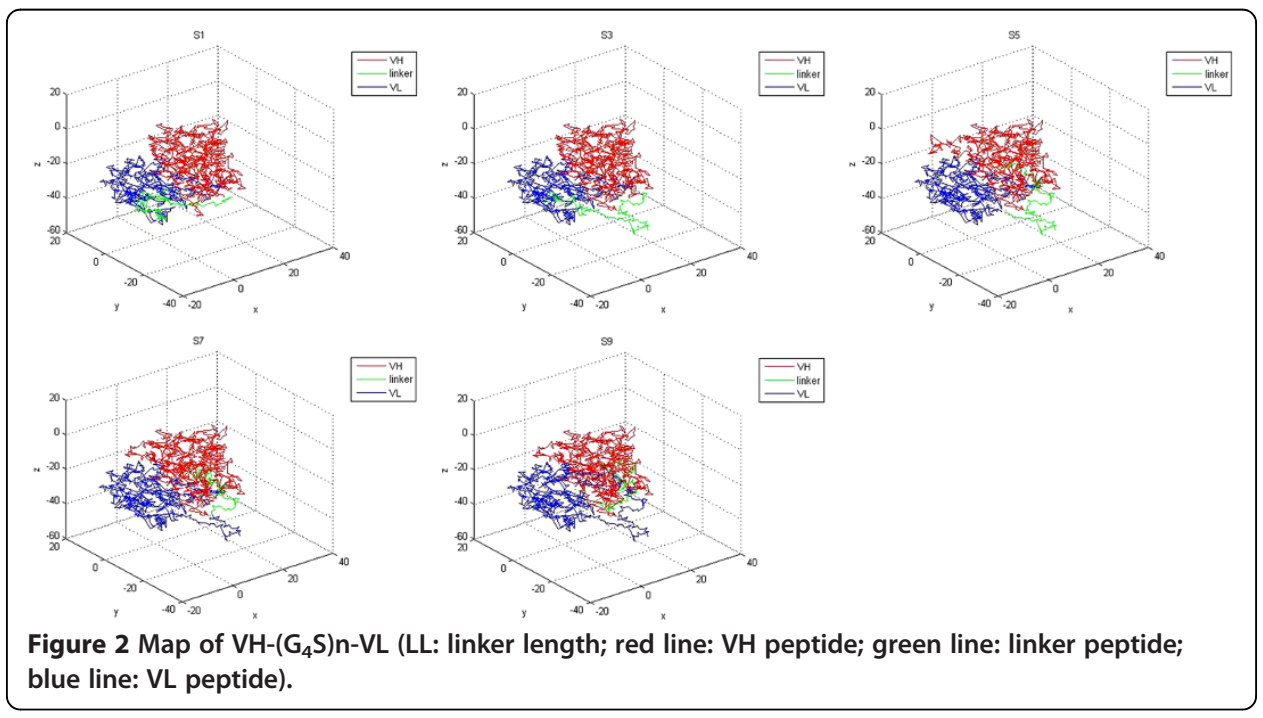


Table 1 Global model accuracy was analyzed by Meta-MQAP

\begin{tabular}{lcr}
\hline Sequence & GDT-TS & RMSD \\
\hline VHL & 64.189 & 2.392 \\
VHL1 & 75.548 & 1.959 \\
VHL2 & 74.893 & 1.920 \\
VHL3 & 64.811 & 2.400 \\
VHL4 & 75.332 & 2.082 \\
VHL5 & 77.544 & 2.016 \\
VHL6 & 75.000 & 2.120 \\
VHL7 & 76.991 & 1.904 \\
VHL8 & 73.783 & 2.229 \\
VHL9 & 60.841 & 2.860 \\
\hline
\end{tabular}

increased within a certain range, the End-to-end distance of VH had relatively large fluctuations. The End-to-end distance of VL basically did not change except when $n=6$ and $n=0$. The data suggests that the major factor for this was that the median value of BC was about 22.6622 in the End-to-end distances of linked peptides. Although the End-to-end distance changes were small, there were fluctuations in the value of $A B$ and $C D$ near the ideal state. Thus, the effects of the linked peptide structural factors $(r)$ on $\mathrm{VH}$ and $\mathrm{VL}$ can be represented in the following equation: $r(n)=$ $\sqrt{\left[A B(n)-\mathbf{A} B_{O}\right]^{2}+\left[C D(n)-C D_{O}\right]^{2}}+\left[B C(n)-B C_{s t}\right]^{2}$. The ideal fusion protein structure should have a stable structure with the linker peptide of minr $(n)$, as shown in Figure 3. The results of $r$ were obtained from the corresponding linker length. The $r$-values were $36.8161,8.0150,0.8415,22.1579,24.4747,582.2451,46.8344,88.6852$, and 112.3846 , with a median value of 24.4747 .

The results suggest that when $n=3$, the $r$-value was the smallest, and the structure of fusion proteins was closest to the ideal state. The $r$-values increased when $n$ increased and hence the linker length increased, in which VH and VL structure would be impacted to a greater extent. When $n$ was 6 , the $r$ value was the most unsatisfactory.

Table 2 The value of $A B \backslash C D \backslash B C$ at different values of $n$

\begin{tabular}{llll}
\hline Sequence & BC & A & CD \\
\hline Wild-type VH & 36.7939 & & \\
Wild-type VL & 14.4022 & & \\
$n=0$ & 29.6393 & 6.8006 & \\
$n=1$ & 16.7822 & 34.9540 & 13.1215 \\
$n=2$ & 22.6653 & 44.7992 & 14.0089 \\
$n=3$ & 22.6622 & 37.5364 & 14.0063 \\
$n=4$ & 19.6630 & 23.6371 & 14.0095 \\
$n=5$ & 23.7909 & 13.59664 & 14.0052 \\
$n=6$ & 46.6356 & 37.536 & 21.8867 \\
$n=7$ & 27.4472 & 12.8588 & 14.0167 \\
$n=8$ & 13.9244 & 24.4632 & 14.0393 \\
$n=9$ & 12.5718 & 26.2316 & 14.0417 \\
\hline
\end{tabular}




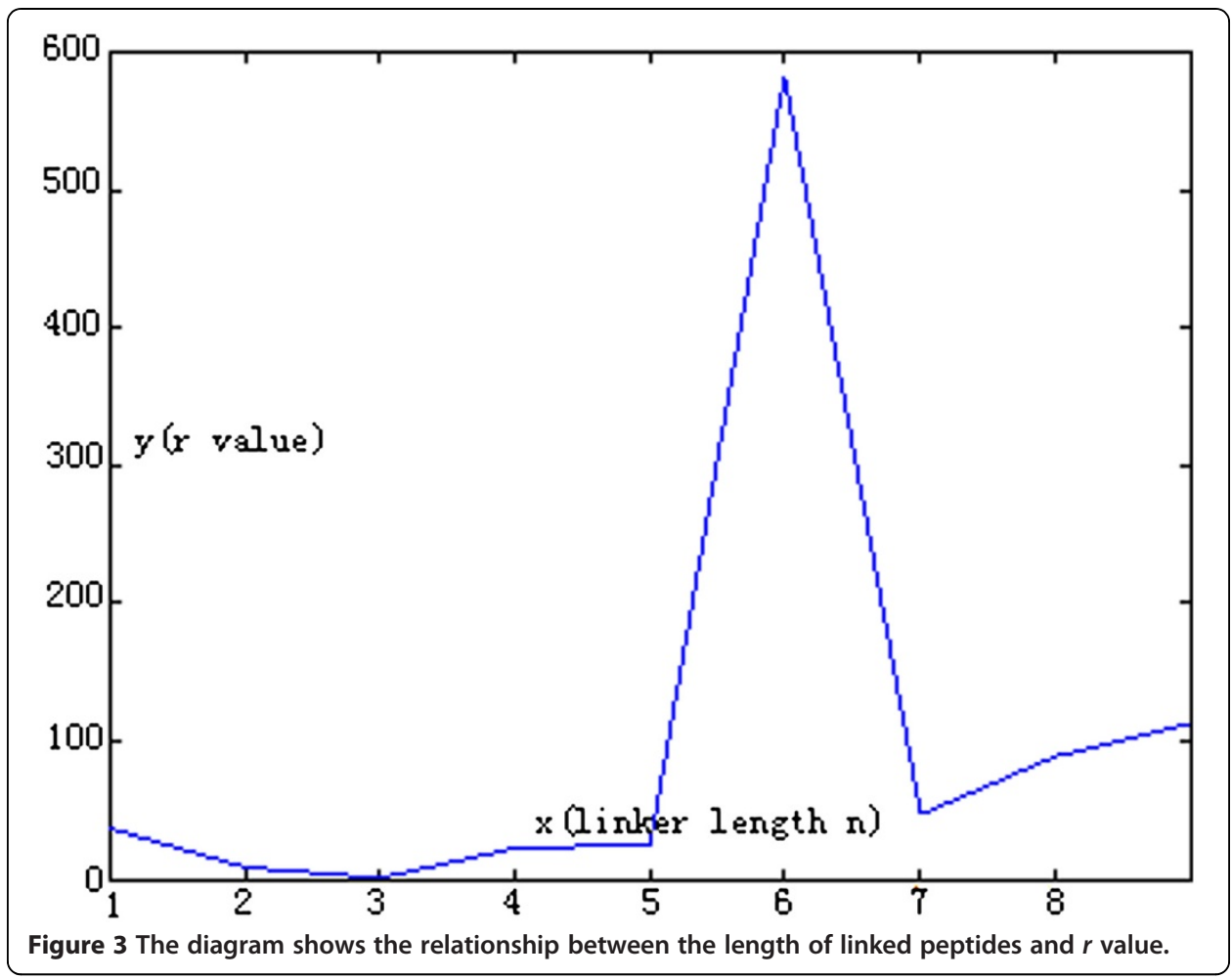

Determination of expression and purity of proteins

Plasmids ND-1scFv-pET28a (+) were transformed into E. coli BL21, and protein expression induced with IPTG. Western blot analysis indicated that BL21 lysates expressed scFv-n proteins with bands of $30 \mathrm{kDa}$ (Figure 4). The sequences encoding the short His-tag peptide were upstream of the multi-cloning site (MCS) of vector pET28a (+), and ND-1scFv-n was expressed as a recombinant fusion protein. Western blot analysis showed that scFv-n protein is expressed in inclusion bodies in the supernatant of BL21 lysates. Inclusion body protein was purified to $94 \%$ by metal affinity chromatography using Ni-NTA resin, which binds to the His-tag protein marker on the $\mathrm{N}$ terminal end of $\mathrm{scFv}$.

\section{Analysis of the relationship between immunoreactivity and End-to-end distance}

The immunoreactivity of purified ND-1scFv-n was determined by ELISA. scFv-n exhibits an immunoreactivity similar to the parental ND-1 antibody, and demonstrated good binding to CCL-187 cells expressing colorectal carcinoma associated antigen LEA. This suggests that scFv-n retains good specificity and activity.

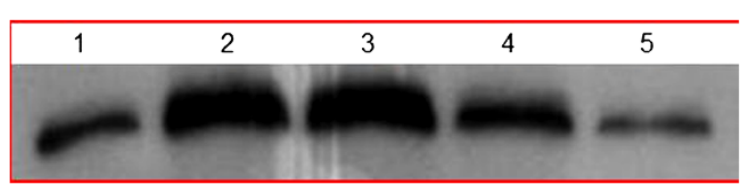

Figure 4 Western blot analysis of ND-1scFv- $\boldsymbol{n}$ in BL21 cells. 1: Expression of pET28a (+)-ND-1scFv with $\left(\mathrm{G}_{4} \mathrm{~S}\right)_{1} ;$; : Expression of pET28a (+)-ND-1 scFv with $\left(\mathrm{G}_{4} \mathrm{~S}\right)_{3} ; 3$ : Expression of pET28a (+)-ND-1scFv with $\left(\mathrm{G}_{4} \mathrm{~S}\right)_{5} ; 4$ : Expression of pET28a (+)-ND-1scFv with $\left(G_{4} S\right)_{7}$; 5 : Expression of pET28a (+)-ND-1scFv with $\left(G_{4} S\right)_{9}$. 
Table 3 shows the relationship between scFv immunoreactivity $\left(\mathrm{A}_{450}\right.$ value) and $r$ values. The immunoreactivity declined with increasing $r$-values. It changed significantly when the $r$-value was less than 42.3716. When the $r$-value exceeded this value, immunoreactivity became relatively stable (Figure 5 ).

\section{Discussion}

Homology modeling has been successfully applied to interpreting the correlation of protein sequence, structure, and function. Using a structural model, multiple sequences of orthologues proteins can be compared and evaluated according to the restrictions of natural selection and requirements of protein folding, stability, dynamics, and function. Homology modeling can help determine which functional groups the protein belongs to based on the analyses of conserved residues in the binding site. Homology modeling also plays an important role in computer-aided drug design [25,26].

One basic issue in the study of protein structure is structural comparison. The relatively direct comparison method is to consider the protein as a rigid structure composed of a series of point sets, then compare the corresponding residues of different proteins. At the beginning, a rigid superposing method was used (to translate and rotate the spatial structure of the protein to find the corresponding residues between two proteins) $[27,28]$. However, Chen proposed using a weight distribution of the atoms composing the protein, and to use this to calculate the protein's gravity center, using a $3 \times 3$ matrix composed of second-order moments [24]. On this basis, one can use principal component analysis (PCA) to find the main and secondary axis. The best rigid superposition is obtained through superposing the gravity centers of the proteins and then rotating them to let their main axes superimpose. In this study, we used the molecular weight of the atoms to get the centric according to the coordinates of each atom.

It is recognized that fusion proteins have varied affinity and anti-tumor activity compared to the original molecules, due in large part to the structural alterations of the fusion proteins [4,28-31]. The inter-peptide linkers can be optimized with computeraided design [32]. Based on homology modeling of derivatives [33], future designs of inter-peptide linkers can be viewed as solving an equation. The structure and characteristics of target molecules, and the composition, length, and flexibility of inter-peptide linker should be taken into consideration [34,35].

In previous studies [35-37], the length and composition of the linkers that have been used to link VH and VL on bivalent single-chain antibody often impact stability and function. Linkers may be too short to fold correctly by intermolecular static influence or be too long to ameliorate the immunogenicity of antibodies. To satisfy these requirements, several design strategies have been developed. One approach is to use the

Table 3 The relationship between $r$-value and the corresponding biology

\begin{tabular}{lll}
\hline $\mathbf{n}$ & $\boldsymbol{r}$ value & A450 value \\
\hline 3 & 0.8415 & 1.17 \\
5 & 24.4747 & 1.02 \\
1 & 36.8161 & 0.82 \\
7 & 46.8344 & 0.75 \\
9 & 112.3846 & 0.71 \\
\hline
\end{tabular}




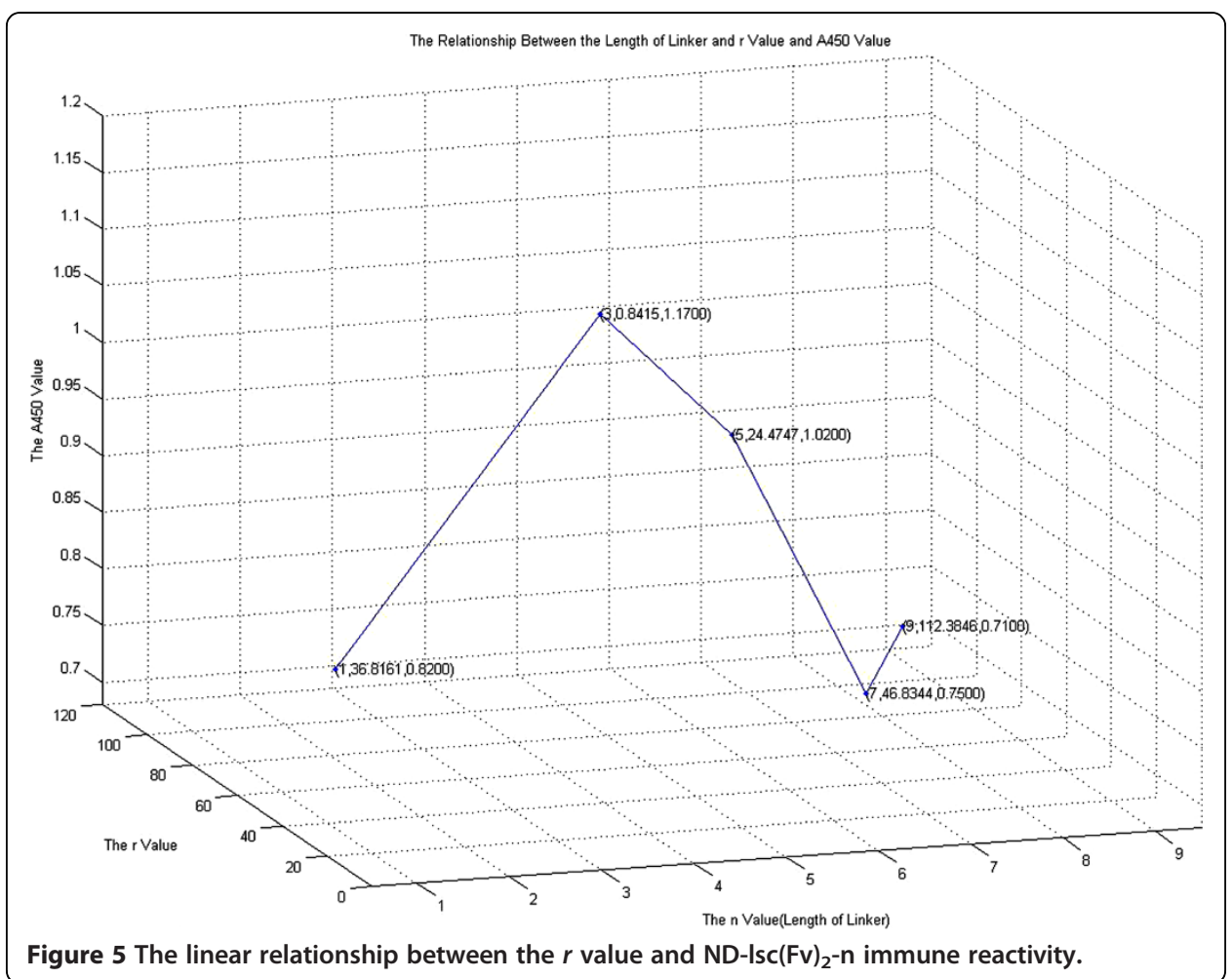

flexible Glycine rich sequences $\left(\mathrm{G}_{4} \mathrm{~S}\right) \mathrm{n}$ as tethers. Linkers comprising repeats of $\mathrm{G}_{4} \mathrm{~S}$ have been used to construct bivalent single-chain antibodies targeting colorectal cancer with linkers of 5-15 amino acids $[18,36]$. With a 5 amino acid linker, immune reactivity was unsatisfactory, possibly because the linker was too short to provide an effective distance for the two antigen-binding sites, which affected the stability of the cross-linked protein. The linker with 15 amino acids tended to fold correctly and retained the bivalent single-chain antibody's affinity and capacity. It has long been noted that sufficient flexibility and length for $\mathrm{VH}$ and $\mathrm{VL}$ domains are achieved by assembling them in the natural $\mathrm{Fv}$ orientation to form a monovalent antigen-binding site, which is comparable to the Fab fragment of native antibodies. It has also been shown that the length and sequence of the linker peptide significantly affects scFv expression and stability [36].

It should be pointed out that the impact of linker length on the activity and affinity of engineered antibodies depends strongly on the distance between the $\mathrm{N}$ - and Cterminal of the $\mathrm{VH}$ domain [37]. A certain degree of flexibility in the linker is required for the functional cooperation of the two subunits. The goal of this study was to characterize novel scFvs and to quantify the impact of linker peptide on binding affinity. Using computer guided homology, scFvs with different linker peptides were proposed based upon the activity and the End-to-end distance. Our aim was to evaluate the impact of $\left(\mathrm{G}_{4} \mathrm{~S}\right) \mathrm{n}$ on the structure and function of $\mathrm{VH}$ and $\mathrm{VL}$, and to find the relationship between VH/VL's End-to-end distance and $n$ (or BC) on bivalent single-chain antibodies targeting colorectal cancer. A multi-factor relationship model was established to evaluate $\mathrm{VH}$ and VL structural factors using the following formula: $r(n)=$ $\sqrt{\left[A B(n)-\mathbf{A} B_{O}\right]^{2}+\left[C D(n)-C D_{O}\right]^{2}}+\left[B C(n)-B C_{s t}\right]^{2}$. Based on simulated data and 
biological experiments, a linear relationship has been established between the immunoreactivity and $r$-values. The immunoreactivity declines as the $r$-value increases. Fusion protein structure is ideal when the $r=3$. When the $n$ value is 6 , protein structure is least satisfactory. However, further exploration of this relationship is needed. Indeed, the expression level and activity of scFv depends largely on the length and sequence of linker. Thus, successful construction of a scFv depends on the selection of a linker that neither interferes with the folding and association of $\mathrm{VH}$ and VL domains nor reduces the stability and recognition abilities of the Fv molecule.

In summary, based on the databases of natural protein structures and their associated functions, we predicted the structure and function of fusion proteins by homology modeling and further conducted biological experiments to validate our calculations. Thus, a dual approach that incorporates molecular modeling and linker design of engineered antibodies with quantitative determination of antibody affinity is useful to optimize construction. Our approach provides not only a rationale for designing novel engineered antibodies using molecular modeling, but also provides new insight into quantifying antibody binding affinity, especially at low protein concentration. A combination of bioinformatics and genetic research may therefore be beneficial in exploring new agents for genetic engineering of antibodies.

\section{Abbreviations \\ scFv: Single-chain Fv antibody; MCS: Multi-cloning site; PCA: Principal component analysis.}

\section{Competing interests}

The author(s) declare that they have no competing interests.

\section{Authors' contributions}

$J H$ Zhang drafted the manuscript. ZG Shang participated in the design of the study and performed the statistical analysis. L Shi conceived of the study, and participated in its design and coordination and helped to draft the manuscript. All authors read and approved the final manuscript.

\section{Acknowledgments}

This research was supported by the natural sciences project plan of the Education Department of Henan province (No. 2010A310018), and the Young Teachers Fund Project of the Institutes of Higher Education of Henan Province (No. 2010GGJS-017).

\section{Author details}

${ }^{1}$ Faculty of Biomedical Engineering of Zhengzhou University, Zhengzhou 450001, Henan Province, People's Republic of China. 'Emergency Department of Xi'an North Hospital, Xi'an 710000, Shaanxi Province, People's Republic of China. ${ }^{3}$ Vascular Endocrinology Department of Xijing Hospital Affiliated to the Fourth Military Medical University, Xi'an 710032, Shaanxi Province, People's Republic of China.

Received: 27 June 2012 Accepted: 17 August 2012

Published: 22 August 2012

References

1. Olafsen T, Sirk SJ, Betting DJ, Kenanova VE, Bauer KB, Ladno W, Raubitschek AA, Timmerman JM, Wu AM: ImmunoPET imaging of B-cell lymphoma using 124I-anti-CD20 scFv dimers (diabodies). Protein Eng Des Sel 2010, 23:243-249.

2. Asano R, Ikoma K, Kawaguchi H, Ishiyama Y, Nakanishi T, Umetsu M, Hayashi H, Katayose Y, Unno M, Kudo T, Kumagai I: Application of the Fc fusion format to generate tag-free bi-specific diabodies. FEBS J 2010, 277:477-487.

3. Fisher AC, DeLisa MP: Efficient isolation of soluble intracellular single-chain antibodies using the twin-arginine translocation machinery. J Mol Biol 2009, 385:299-311.

4. Geng SS, Feng JN, Li Y, Sun Y, Gu X, Huang Y, Wang Y, Kang X, Chang H, Shen B: Binding activity difference of anti-CD20 scFv-Fc fusion protein derived from variable domain exchange. Cell Mol Immunol 2006, 3:439-443.

5. Muller D, Karle A, Meissburger B, Höfig I, Stork R, Kontermann RE: Improved pharmacokinetics of recombinant bispecific antibody molecules by fusion to human serum albumin. J Biol Chem 2007, 282:12650-12260.

6. Stone E, Hirama T, Tanha J, Tong-Sevinc H, Li S, MacKenzie CR, Zhang J: The assembly of single domain antibodies into bispecific decavalent molecules. J Immunol Methods 2007, 318:88-94.

7. Guo JQ, Li QM, Zhou JY, Zhang GP, Yang YY, Xing GX, Zhao D, You SY, Zhang CY: Efficient recovery of the functional IP10-scFv fusion protein from inclusion bodies with an on-column refolding system. Protein Expr Purif 2006, 45:168-174. 
8. Shan DM, Press OW, Tsu TT, Hayden MS, Ledbetter JA: Characterization of scFv-lg constructs generated from the anti-CD20 mAb 1F5 using linker peptides of varying lengths. J Immunol 1999, 162:6589-6595.

9. Shen Z, Yan H, Zhang Y, Mernaugh RL, Zeng X: Engineering peptide linkers for scFv immunosensors. Anal Chem 2008, 80:1910-1917.

10. James BG, Leisha SM, Frank MR: Effect of linker sequence on the stability of circularly permtuted variants of ribonuclease T1. Bioorganic Chemistry 2003, 31:412-424.

11. Arcangeli C, Cantale C, Galeffi P, Gianese G, Paparcone R, Rosato V: Understanding structural/functional properties of immunoconjugates for cancer therapy by computational approaches. J Biomol Struct Dyn 2008, 26:35-48.

12. Wajanarogana $S$, Prasomrothanakul $T$, Udomsangpetch $R$, Tungpradabkul $S$ : Construction of a human functional single-chain variable fragment ( $\mathrm{scFv}$ ) antibody recognizing the malaria parasite Plasmodium falciparum. Biotechnol Appl Biochem 2006, 44:55-61.

13. Clark KR, Walsh ST: Crystal structure of a $3 B 3$ variant-a broadly neutralizing HIV-1 scFv antibody. Protein SC 2009, 18:2429-2441.

14. Kamphausen S, Holtge N, Wirsching F, Morys-Wortmann C, Riester D, Goetz R, Thürk M, Schwienhorst A: Genetic algorithm for the design of molecules with desired properties. J Comput Aided Mol Des 2002, 16:551-567.

15. Hajduk PJ, Huth JR, Tse C: Predicting protein druggability. Drug Discov Today 2005, 10:1675-1682.

16. Jiang $Z$, Zhou $Y$ : Using bioinformatics for drug target identification from the genome. Am J Pharmacogenomics 2005, 5:387-396.

17. Kim GB, Wang Z, Liu YY, Stavrou S, Mathias A: Goodwin K.J, Thomas JM, Neville DM: A fold-back single-chain diabody format enhances the bioactivity of an anti-monkey CD3 recombinant diphtheria toxin-based immunotoxin. Protein Eng Des Sel 2007, 20:425-432.

18. Fang J, Jin HB, Song JD: Construction, expression and tumor targeting of a single-chain Fv against human colorectal carcinoma. World J Gastroenterol 2003, 9:726-730.

19. Arnold K, Bordoli L, Kopp J, Schwede T: The SWISS-MODEL Workspace: A web-based environment for protein structure homology modelling. Bioinformatics 2006, 22:195-201.

20. Schwede T, Kopp J, Guex N, Peitsch MC: SWISS-MODEL: an automated protein homology-modeling server. Nucleic Acids Res 2003, 31:3381-3385.

21. Guex N, Peitsch MC: SWISS-MODEL and the Swiss-PdbViewer: An environment for comparative protein modelling. Electrophoresis 1997, 18:2714-2723.

22. McGuffin L: Benchmarking consensus model quality assessment for protein fold recognition. BMC Bioinforma 2007, 8:345.

23. Pawlowski M, Gajda MJ, Matlak R, Bujnicki JM: MetaMQAP: A meta-server for the quality assessment of protein models. BMC Bioinforma 2008, 9:403.

24. Chen SC, Chen TH: Retrieval of 3D protein structures[C]. New York: ICIP: Soifer Proceedings of the 2002 International Conference on Image Processing (ICIP 2002); 2002:933-936.

25. Szantai-Kis C, Kovesdi I, Eros D, Nanhegyi P, Ullrich A, Orfi L: Prediction oriented QSAR modelling of EGFR inhibition. Curr Med Chem 2006, 13:277-287.

26. Le Gall F, Reusch U, Little M, Kipriyanov: Effect of linker sequences between the antibody variable domains on the formation, stability and biological activity of a bispecific tandem diabody. Protein Eng Des Sel 2004, 17:357-366.

27. Taylor WR: Protein structure comparison using iterated double dynamic programming. Protein Sci 1999, 8:654-665.

28. Chen Q, Zhou MQ, Suo Q, Wang Y: Protein three-dimensional space unified coordinate system established. Journal of Northwest University (Natural Science Edition) 2007, 37:205-207.

29. Tranchant I, Hervé AC, Carlisle S, Lowe P, Slevin CJ, Forssten C, Dilleen J, Bhalla R, Williams DE, Tabor AB, Hailes HC: Design and synthesis of ferrocene probe molecules for detection by electrochemical methods. Bioconjug Chem 2006, 17:1256-1264.

30. Bello-Rivero I, Torrez-Ruiz Y, Blanco-Garcés E, Pentón-Rol G, Fernández-Batista O, Javier-González L, GerónimoPerez H, López-Saura P: Construction, purification, and characterization of a chimeric TH1 antagonist. BMC Biotechnol 2006, 6:25.

31. Shibata K, Maruyama-Takahashi K, Yamasaki M, Hirayama N: G-CSF receptor-binding cyclic peptides designed with artificial amino-acid linkers. Biochem Biophys Res Commun 2006, 341:483-488.

32. Zou BJ, Zhang Q, Liang LM: Similarity Comparison of Protein Structures via Protein Space Partition in Spherical Polar Coordinates. Journal of Cmputer-aided Design \& Computer Graphics 2009, 21:205-207.

33. Zhang JH, Yun J, Shang ZG, Zhang XH, Pan BR: Design and optimization of a linker for fusion protein construction. Prog Nat Sci 2009, 19:1197-1200

34. Fang M, Jiang X, Yang Z, Yu XH, Yin CC, Li H, Zhao R, Zhang Z, Lin Q, Huang HL: Effects of inter-peptide linkers to the biological activities of bispecific antibodies. Chin Sci Bull 2003, 48:1912-1918.

35. Ying L, Chen JH, Zhang XG: Research Progress in the Linker of Fusion Protein. Biotechnology 2008, 18:92-94

36. Yan DD, Fang J, Song JD: Construction and Expression of Bivalent Single-chain Antibodies with Different Linker Sequence against Human Colorectal Carcinoma. Chinese Journal of Cell Biology 2007, 29:272-276.

37. Gu X, Jia XL, Feng JN, Shen BF, Huang Y, Geng SS, Sun YX, Wang YG, Li Y, Long M: Molecular Modeling and Affinity Determination of scFv Antibody: Proper Linker Peptide Enhances Its Activity. Ann Biomed Eng 2010, 38:537-549.

doi:10.1186/1742-4682-9-38

Cite this article as: Zhang et al: Analysis of the relationship between end-to-end distance and activity of singlechain antibody against colorectal carcinoma. Theoretical Biology and Medical Modelling 2012 9:38. 